\title{
OBSERVATIONS ON PHOTICALLY EVOKED OCCIPITAL AND VERTEX WAVES DURING SLEEP IN MAN*
}

\author{
K. A. Kooi, B. K. Bagchi, R. N. Jordan \\ Neuropsychiatric Institute, University of Michigan, Ann Arbor, Mich.
}

\section{Introduction}

Information about the character of the visual evoked response in man during sleep and possible differences between waking and sleep is as yet limited. Vanzulli et al. (1960) reported that response latencies were increased and "positive waves" augmented. Cigánek (1961) noted that the early triphasic complex was unchanged except for increased latency while a later wave ("V") was increased in amplitude, broadened and delayed. He observed typical K-complexes during deep sleep. Guillard (1960) has reported that an anteriorly distributed response may persist in sleep when the posterior response has disappeared. He also reported longer response latency as depth of sleep increased. Brazier (1960) and Barlow (1960) have averaged occipital responses to flash in a subject both awake and asleep and have shown that periodic alpha range oscillations may disappear during sleep.

The present communication describes morphological characteristics of cerebral responses evoked by single intense photic stimuli presented during sleep, compares these characteristics with those observed in waking and relates response morphology to electrographic and behavioral measures of level of consciousness. Attention is also given to topographical features of the responses, particularly in relation to distinctions that may be drawn between central and occipital events.

\section{Method}

Characteristics of the recording system have been previously described (Kooi and Bagchi, 1963). Averaging was accomplished with the Mnemotron CAT, generally with four inputs and dwell times of 2.5 or $5 \mathrm{msec}$. The averaged responses were recorded through one pen unit of a Grass Model IV-BS electroencephalograph. Both the computer static display and the EEG write-out were linear from $2-35 \mathrm{c} / \mathrm{sec}$. with reduction to 40 per cent of linear range amplitude at $100 \mathrm{c} / \mathrm{sec}$. Latency data was obtained directly from the static display. Upper frequency filtering, used to reduce muscle artifact distortion, caused an average phase lag of three msec. with a range of 1 to 5 msec.

From 400 to 2000 responses were averaged in each of seven subjects during thirty-five experimental sessions devoted exclusively to recording responses during sleep. $\Lambda$ n almost equal number of experiments was

- This study was supported in part by Grant NB-02560, National Institute of Neurological Diseases and Blindness, National Institutes of Health, Public Health Service, Bethesda, Md. 
devoted to the study of technical factors, artifacts, both instrumental and physiological and effects of altering stimulus parameters in the alert subject. Most of the sleep records were obtained after the subjects had been mildly sleep deprived ( $0-3$ hours sleep during previous 24 hours). Subjects were dark adapted for at least 30 minutes. Pupils were maximally dilated with 10 per cent neosynephrine in three subjects during one experiment each.

The photic stimulus was presented randomly once every 8-10 seconds with a Grass PS2 photo-stimulator. The relatively long time interval was chosen for three reasons: (1) to minimize light adaptation (Mahneke, $1957) ;(2)$ to allow for recovery of vertex sharp wave mechanisms; and (3) to avoid arousing the subject. The face of the flash lamp was $10 \mathrm{~cm}$. from the subject's closed eyes. The lamp was encased in a double-walled box to eliminate subjective awareness of the click associated with discharge of the tube. The basic data was collected with a lamp intensity rated at 50,000 peak candles.

For statistical comparisons, the responses were averaged in groups of 10. This procedure had the advantage of providing information about response variation and permitting collection of homogeneous samples in reference to level of sleep. Larger samples were recorded for visual comparisons.

Sleep levels were classified electrographically according to the schema of Loomis, Harvey and Hobart (1937), A-waking, B-drowsiness, C \& Dlight and moderately deep sleep and E-deep sleep.

Reaction times were obtained with an electromechanical transducer attached to the index finger of the left hand.

Retinal, ocular movement, and periorbital muscle potentials were observed to spread widely over the anterior portion of the scalp and face. Retinal responses continued to be evident during sleep whereas ocular movement and reflex muscle responses were no longer detectable. Studies utilizing linear arrays of electrodes arranged about the eye indicated that the $\mathrm{b}$-wave of the scotopic electroretinogram was recorded as a positive wave of $20-40 \mu \mathrm{V}$ with a latency of $70-80 \mathrm{msec}$. immediately above and below the eye, decreasing to $3-5 \mu \mathrm{V}$ at a chin lead $12 \mathrm{~cm}$. distant. A similar amplitude gradient was observable over the scalp although precise measurements were not possible because of the superimposition of cerebral responses. The amplitude of the b-wave was appreciably less (10$15 \mu \mathrm{V}$ ) posterior to the outer canthus than above or below the eye. In some subjects a-waves were also detectable from these regions. Two subjects (S2, S6) had active blink reflexes during waking. Periorbital muscle twitch responses were recognized as sharp rapid deflections occurring singly or in a cluster beginning approximately $50 \mathrm{msec}$. after the stimulus. In the subject with the greatest myoclonic response, phase of the individual deflections was consistently inverted above and below the eye. A late wave (200-250 msec.), related to ocular rotation and surface positive above the orbit, was also present in these two individuals.

A noncephalic reference (Stephenson and Gibbs, 1949) was routinely 
employed. An ear-noncephalic lead pair served to monitor extracerebral sources of artifact.

\section{Results}

All subjects had active multiphasic responses during waking and at all levels of sleep. In some subjects, the general pattern of the occipital response appeared little changed, individual components appearing to be common to both waking and sleep. In other subjects, the form changed greatly and it was not immediately apparent whether the alterations were attributable to increasing or decreasing amplitudes of waking events or to the emergence of qualitatively different features.

The amplitude of surface negative activity in the 60 to $100 \mathrm{msec}$. latency range was decreased in six of the seven subjects, (examples in FIGURES 1, 2, and 3); no change was detected in the seventh (S7). This finding was examined statistically in one subject (S1) in which the trough and peak latencies of this wave were virtually identical for waking and sleep, utilizing measurements of averaged responses gathered in groups of 10 during five experimental sessions. The amplitude reduction was significant both for those responses recorded during drowsiness $(t=4.4$, $p<.001)$ and sleep $(t=6.6, p<.001)$. In five individuals a prominent late negative wave developed concomitantly with attenuation of the earlier negative activity as the subject fell asleep (FIGUREs 1, 3, and 4).

Vertex sharp waves, culminating between $130-160 \mathrm{msec}$., were readily identified in six subjects. Their amplitudes were decreased during sleep in all cases. This wave could not be established with certainty in the seventh subject (S7). In contrast to the behavior of the vertex sharp wave, an earlier surface negative event (90-110 msec.) appeared, either as a new wave or as an augmentation of a wave with similar latency during waking, with the onset of sleep in six of the subjects (not identified in S6). This event was not necessarily synchronous with the occipitoparietal wave commonly present within this same general latency range (FIGURE 5).

At deeper levels of sleep, response patterns in central and occipital regions tended to become more alike. Occasionally, they were observed to be highly similar. The degree of similarity was enhanced by faster rates of stimulation, i.e., $1 / \mathrm{sec}$. as compared with $1 / 8-10 \mathrm{sec}$.

Average latencies of major deflections were calculated for all subjects from data gathered at one light intensity. Dominant level of sleep during the computation of each average was recorded and latency scores were grouped according to whether the EEG pattern indicated waking, drowsiness, light or moderately deep sleep or very deep slcep. The results are presented in FIGURE 5. In general, latencies gradually increased in both occipital and central regions as depth of sleep increased, the increments being greater for later events. It is noteworthy, however, that instances were observed where the latency was not increased (S1, S2, S5) and 


\section{ALTERATION OF OCCIPITAL EVOKED RESPONSE ASSOCIATED WITH ONSET OF SLEEP}

EVOKED

RESPONSE

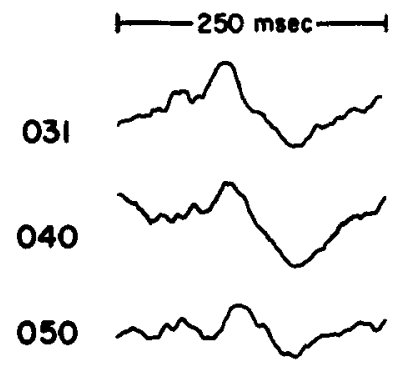

062



074

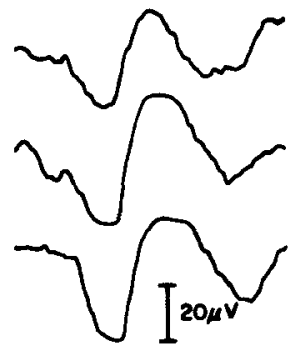

$\begin{array}{cccccc}1 & 1 & 1 & 1 & 1 & 1 \\ 0 & 20 & 40 & 60 & 80 & 100\end{array}$
SPONTANEOUS REACTIVITY REACTION TIME

PATTERN

(sec)

a-blocking

.25

a, $\beta$

a-blocking

.25

$\theta$

$a-$ roturn

.35

$\theta$

a-return

.45

$\theta$

a-rofurn

$.65(9 / 10)$

contral di-

phasics and spindles

central diphasics and spindles

\section{K-complex} $(2 / 10)$

$.85(1 / 10)$

K-complex $(3 / 10)$

no reaction

FIGURE 1. Progressive alteration of the occipital visual evoked response during the onset of sleep. Each curve is the average of 10 responses, recorded sequentially over a 25 minute period. Note inversion of early phase of the response and development of 40 microvolt late surface negative event. Changes occurring in the spontaneous EEG pattern, its reactivity at the instant of the stimulus and the subject's responsiveness are also tabulated. Sheet nos. 031-100. 


\section{ALTERATION OF OCCIPITAL EVOKED RESPONSE ASSOCIATED WITH DROWSINESS AND SLEEP}

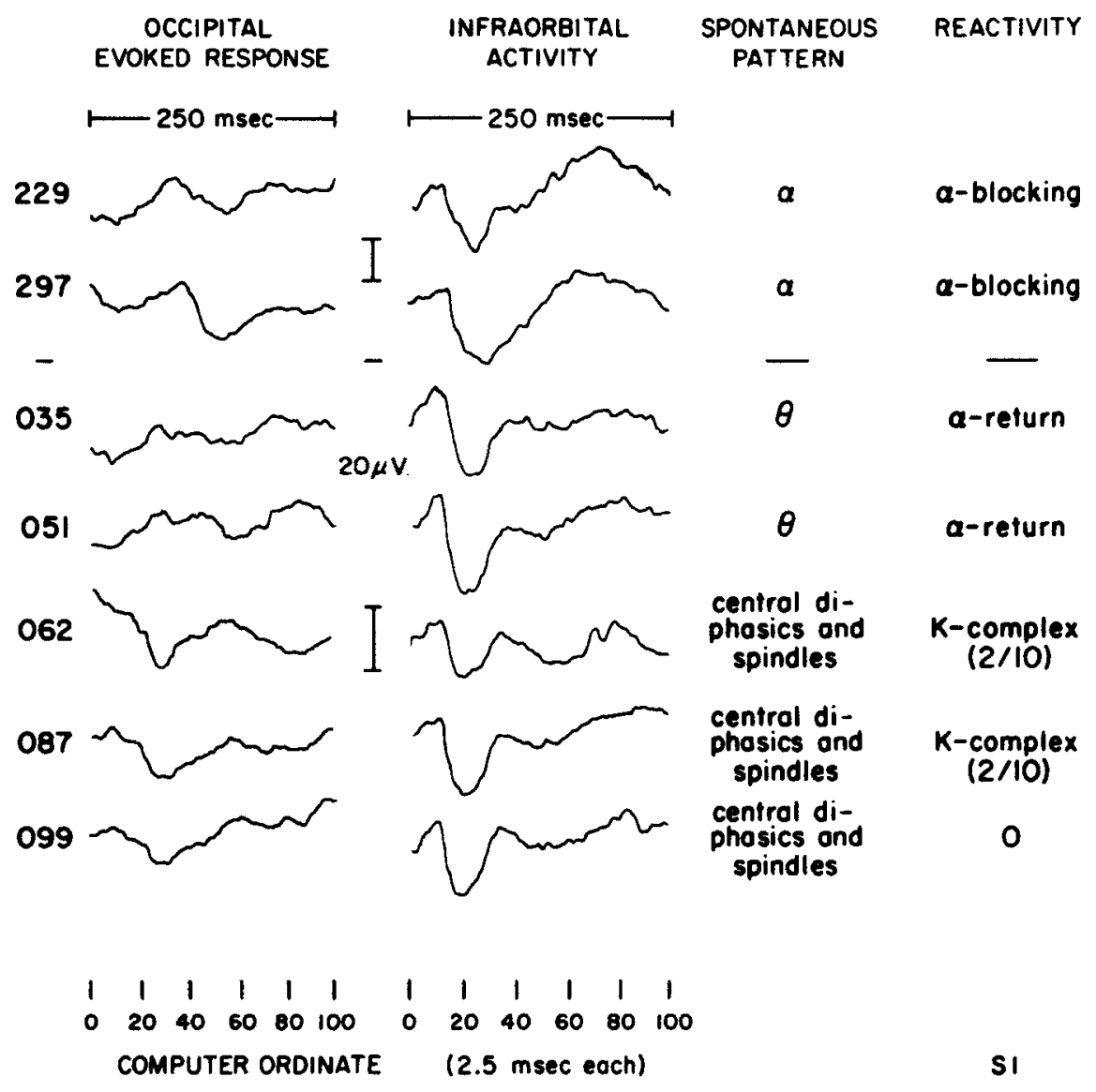

Figure 2. Comparison of the visual evoked response during waking, drowsiness and light sleep in a subject with maximally dilated pupils. Each curve is the average of 10 responses. The samples obtained during drowsiness and light sleep were obtained successively over a $23 \mathrm{~min}$. period. The waking curves were obtained following sleep in this subject. The findings parallel those recorded in S2. The scotopic electroretinogram as recorded by an infra-orbital electrode remained well developed during drowsiness and light sleep. Reaction times were not obtained during this experiment. Waking229, 297; drowsiness-035, 051; light sleep-062, 087, 099. 


\section{OCCIPITAL EVOKED RESPONSE \\ PEAK LATENCIES OF DEFLECTIONS $>2 \mu \mathrm{V}$ \\ (420 responses summoted in groups of 10 )}

$250 \mathrm{msec}$

AWAKE

\$3
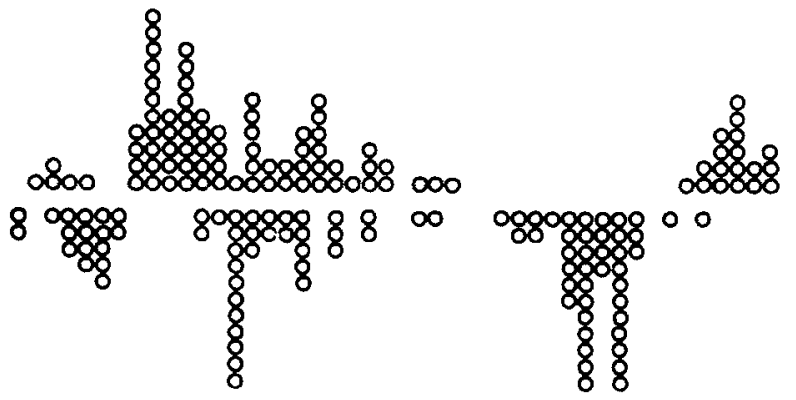

ASLEEP

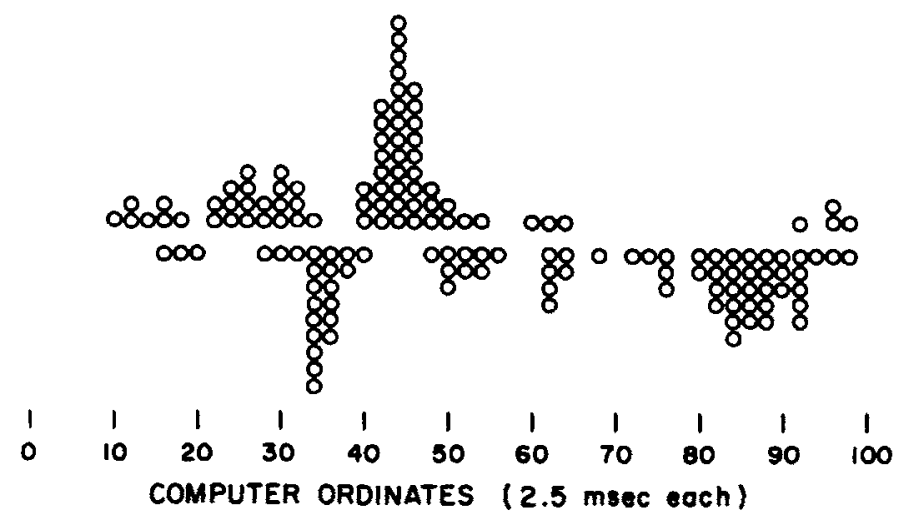

Figune 3. Each point represents the latency of a deflection greater than two microvolts. Four hundred and twenty responses were averaged in groups of 10 both during waking and sleep. See text. 


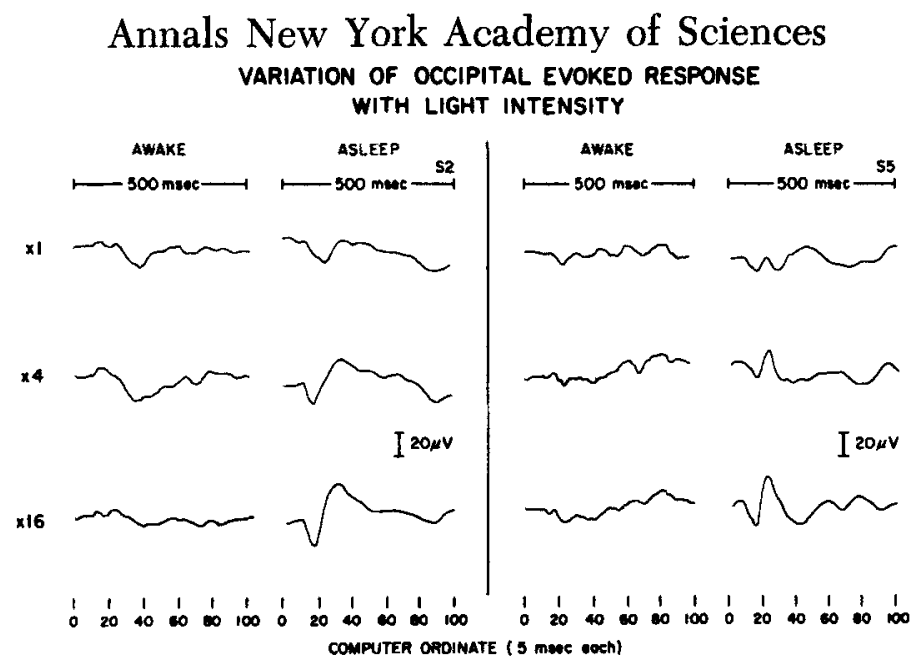

Figure 4. Averages of 50 responses to three different light intensities awake and asleep. Analysis time 500 msec. Overall response amplitude was not appreciably changed by increased light intensity within this range in the alert subject whereas an effect was readily apparent when the subject was sleeping. Amplitude was higher with the lowest light intensity asleep than with the highest intensity awake.

even, rarely, slightly decreased ( $\mathrm{S} 1, \mathrm{~S} 4$ ). The data illustrated in FIGURE 3 offers additional evidence in support of these conclusions.

Examination of responses at different light intensities revealed that this factor was an important determinant of wave form (Figures 4 and 6). Increased brightness led to increase in the amplitude of the late negative wave as well as decrease in latency of both the major positive and negative peaks. The shortening of latency with each fourfold increase in light intensity was in the order of 10-15 msec. Another effect could be observed repeatedly in some subjects. This was characterized by the gradual recession of a surface negative wave in the latency range of the vertex sharp wave concomitant with the emergence of an earlier surface negative event.

All subjects were required to react to the stimulus by movement of the left index finger as long as they retained consciousness. Average reaction times (per 10 trials) gradually slowed as the subject became drowsy, paralleling changes both in resting EEG patterns and average response patterns. These alterations are exemplified in FIGURE 1 . EEG reactivity was also characteristic at each level. No behavioral responses occurred without evidence of change of electrical patterns. K-complexes were observed in the absence of behavioral response.

Rapid habituation of the K-complex response to the relatively intense light was noted in all subjects. These responses were largely limited to the first few presentations of the light during the initial appearance of light sleep. That the habituation was specific for the experimental stimulus was indicated by the observation that they could be readily evoked by low intensity stimuli of other modalities. 


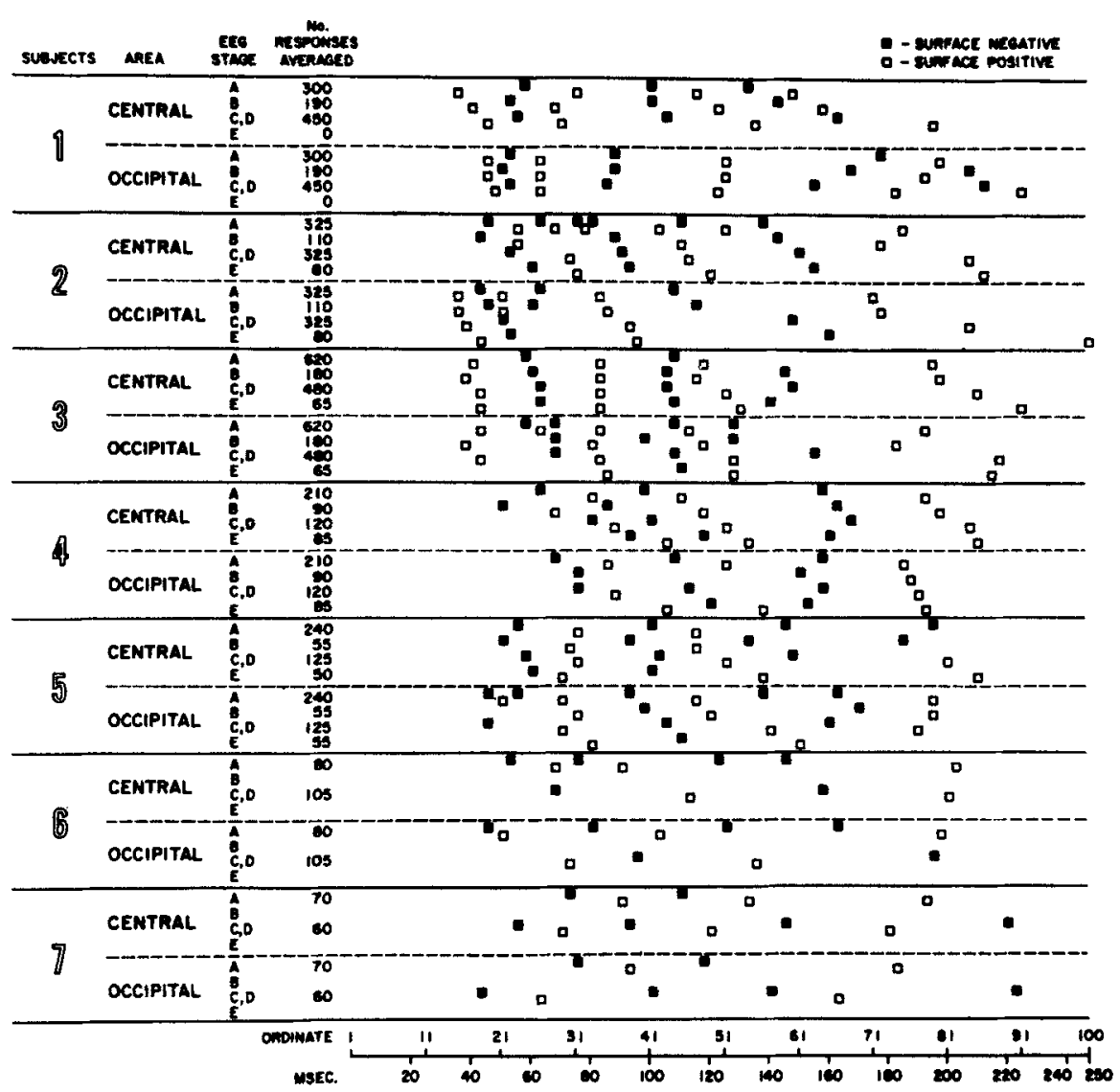

Figure 5. Visual evoked responses during waking and sleep. Latency comparisons.

\section{Discussion}

As Cobb and Dawson (1960) have pointed out, the conditions of the examination must receive more than cursory attention in the analysis of the characteristics of the averaged response. Such factors as sites of "active" and "reference" electrodes, intensity of the stimulus and its rate of presentation affect the form of the recorded event and may actually determine whether or not a particular wave is identified in a given subject. Increasing light intensity will shorten initial inflection and peak latencies and increase the magnitude of certain events. It also may result in the emergence of new waves concurrently with attenuation of events that are well developed at a lower stimulus intensity. The mechanism of this process remains to be determined.

Interpretation of the changes in response configuration observed during sleep requires consideration of ocular factors, such as pupil size, eyeball 


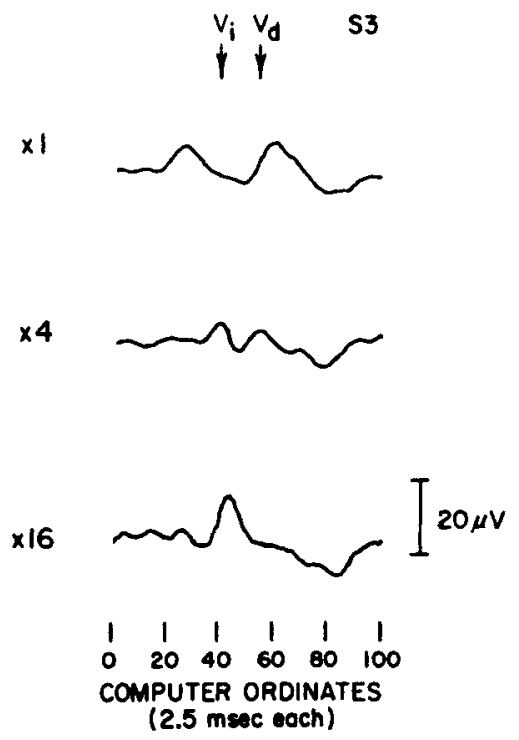

Figure 6. Illustration of a common effect of increased stimulus intensity: attenuation of amplitude of one or more waves with augmentation of another. Average of 25 responses each. Selected to be representative of intensity data showing "paradoxical intensity effect."

position, and level of retinal excitability, that might affect neural activity prior to its relay to the lateral geniculate body. Naquet et al. (1960) have demonstrated that pupil size affects the potentials recorded from chiasm and lateral geniculate but noted considerable independence at the cortical level. Our studies in which the subjects' eyes were maximally dilated and a wide range of intensities employed indicate that the waking-sleep differences are not due solely to pupillary miosis. The effects of globe position and afferent retinal connections cannot be adequately estimated in man.

It is a question of more than semantic interest to inquire whether the alterations occurring with sleep are specifically associated with the general lowering of the level of vigilance or reflect habituation to the stimulus. We believe that the changes of the occipital response observed in this study are not appropriately described as habituation because of these considerations: (1) no significant change in the character of the response occurs without a change in the level of consciousness, i.e., the response during waking is essentially the same during later series, after presentation of several hundred stimuli, as during early series; this is also true (although form is changed from waking) during sleep; (2) the described changes in pattern occur concomitantly with the appearance of spon- 
taneous rhythms indicating onset of drowsiness and sleep; and (3) the characteristic change associated with electrical habituation is attenuation of the response whereas during sleep both augmentation and attenuation may occur.

The process of habituation, however, appears to be active in two other situations: (1) in relation to gradual reduction of the height of the vertex sharp wave over the first few series of stimuli during waking and (2) in relation to the rapid disappearance of the K-complex during sleep. In these cases, a change occurs in the nature of the cerebral response while the general level of vigilance remains relatively constant.

The amplitude reduction of the early occipital response in sleep when spontaneous brain waves are high in amplitude, augmentation of late response amplitude and widening of its wavelength in addition to phase change compared with waking need to be explained on the basis of known anatomico-physiological considerations. Evidently this is difficult at the present state of knowledge. However, it is tempting to speculate about factors which may have bearing on the above-mentioned changes.

If one is permitted to draw an inference that in sleep, the barrage of discharges from reticular formation to cortex is diminished, contrary to waking, the total excitation level of the brain milieu in the former state will be lessened. If then the light stimulus strikes the visual system, the response, because of nonsupport from this deficit milieu, will suffer by that much initially (say within 80 or so milliseconds). But the situation cannot remain the same after the initial onslaught (say after 80 or so milliseconds). The intrusion by light can be assumed after this time lag to somewhat heighten the excitation level. Because by this time there will be manifest disturbance of interrelated electrical fields and subthreshold cooperation of reticular formation-which of course may be short of causing behavioral arousal. During sleep single light stimulus is given every 8 to 10 seconds so the collapsing of the interacting fields and withdrawal of the influence of reticular formation will also be assured in the interval.

This description cannot take into cognizance the perpetual problem of analysis of complex biological waves. For instance one would like to know in the above situation what part the constituent neural units play in emphasizing a particular sign (positive or negative), in giving high amplitude to one short wave and low amplitude to another, high amplitude to one long wave and low amplitude to another, even though we assume such operant principles as spatial summation, temporal dispersion, phase cancellation, and balancing of multisynaptic facilitoryinhibitory influences.

\section{Summary}

Cerebral responses to light were consistently altered during sleep. The occiput negative phase observed at $60-100 \mathrm{msec}$. during waking was typically reduced in amplitude and, in some subjects, a positive deflec- 
tion developed within this latency range. Augmentation of a later negative event occurred in most of the subjects. Latencies of late components were usually increased while those of early deflections were not appreciably changed. The exact form of the response was found to be related to stimulus intensity and individual components were observed to be either increased or decreased in magnitude as the light was made brighter. This "paradoxical intensity effect" appeared of particular importance in analyzing two components of the vertex sharp wave.

\section{Acknowledgment}

We wish to express our appreciation to Wendel Wickland for recording and tabulating portions of the data presented.

\section{References}

BarLow, J. S. 1960. Rhythmic activity induced by photic stimulation in relation to intrinsic alpha activity of the brain in man. Electroencephalog. Clin. Neurophysiol. 12: $317-326$.

Brazier, M. A. B. 1960. Long-persisting electrical traces in the brain of man and their possible relationship to higher nervous activity. Electroencephalog. Clin. Neurophysiol. Suppl. 13: 347-356.

CIGÁNEK, L. 1961. The EEG response (evoked potential) to light stimulus in man. Electroencephalog. Clin. Neurophysiol. 13: 165-172.

CobB, W. A. \& G. D. DAwson. 1960. The latency and form in man of the occipital potentials evoked by bright flashes. J. Physiol. 152: 108-121.

Gurllard, A. 1960. La réactivité au cours du sommeil physiologique de l'homme. Thèse de Médecine. Foulon, Paris, France.

Koor, K. A. \& B. K. BAGCHI. 1963. Visual evoked responses in man: Normative data. This Annals.

Loomis, A. L., E. N. Harvey \& G. A. Hobart. 1937. Cerebral states during sleep, as studied by human brain potentials. J. Exptl. Psychol. 21: 127-144.

MahNEKE, A. 1957. Electroretinography with double flashes. Acta. Ophth. 35: 131-141.

Naquet, R., H. Regis, M. Fischer-Williams \& A. Fernández-Guardiola. 1960. Variations in the responses evoked by light along the specific pathways. Brain. 83: $52-56$.

Stephenson, W. \& F. A. Grbbs. 1949. Electroencephalograms recorded with noncephalic electrodes as a reference. Electroencephalog. Clin. Neurophysiol. 1: 523.

Vanzulli, A., J. Bogacz, P. Handler \& E. García AustT. 1960. Evoked responses in man. I. Photic responses. Acta Neurol. Latinoamer. 6: 219-231. 